\title{
Delayed Hypersensitivity Reaction to Lenalidomide: 2 Different Clinical Patterns in the Same Patient
}

\author{
Noguerado-Mellado $\mathrm{B}^{1,3}$, Rojas-Pérez-Ezquerra $\mathrm{PD}^{1,3}$, Tornero \\ Molina $\mathrm{P}^{1,3}$, Ballesteros $\mathrm{M}^{2,3}$, Zubeldia Ortuño JM $\mathrm{M}^{1,3}$ \\ ${ }^{1}$ Allergy Department, Hospital General Universitario Gregorio \\ Marañón, Madrid, Spain \\ ${ }^{2}$ Haematology Department, Hospital General Universitario \\ Gregorio Marañón, Madrid, Spain \\ ${ }^{3}$ Gregorio Marañón Health Research Institute, Madrid, Spain
}

J Investig Allergol Clin Immunol 2019; Vol. 29(4): 309-311 doi: $10.18176 /$ jiaci.0386

Key words: Delayed. Desensitization. Hypersensitivity. Lenalidomide. Skin tests.

Palabras clave: Desensibilización. Hipersensibilidad. Lenalidomida. Pruebas cutáneas. Retardadas.

Lenalidomide is an immunomodulatory and antiangiogenic agent approved for the treatment of oncological and inflammatory diseases, such as multiple myeloma, myelodysplastic syndrome (MDS), and mantle cell lymphoma. It is administered alone or with dexamethasone in relapsed and refractory multiple myeloma or as maintenance therapy following autologous stem cell transplantation [1].

Lenalidomide has been associated with cutaneous eruptions [2]. Lowering the dose to prevent cutaneous reactions is ineffective $[3,4]$. Skin reactions have been described in approximately $21 \%$ of patients and are the most frequent adverse event with this drug [5].

Truly allergic reactions are difficult to distinguish from toxic adverse effects in patients receiving lenalidomide. Immediate and delayed hypersensitivity reactions are rare, with only a few cases reported, most of which did not involve a complete allergological work-up [6]. Hypersensitivity reactions include urticaria, exanthema, drug reaction with eosinophilia and systemic symptoms syndrome, StevensJohnson syndrome, and toxic epidermal necrolysis $[2,3,7]$.

We report a case involving both skin toxicity and a true delayed hypersensitivity reaction to lenalidomide.

The patient was a 78-year-old man with a history of prostate cancer in 2012 in complete remission and current del (5q) MDS type RAEB 1. He started treatment with $10 \mathrm{mg}$ of lenalidomide for 21 days every 28 days. Following the second cycle, 3 days after the dose, he developed erythematous maculopapular exanthema on the thorax, back, and limbs, with no mucosal or systemic involvement. Oral corticosteroids were administered, and the reaction resolved within 15 days. Lenalidomide was discontinued, and he was referred to our allergy department. After signing an informed consent document, the patient underwent patch tests with lenalidomide at $10 \%$ in dimethyl sulfoxide (DMSO), and readings at day 2 and day 4 were negative. We performed patch tests with lenalidomide in DMSO because it is a useful vehicle that 


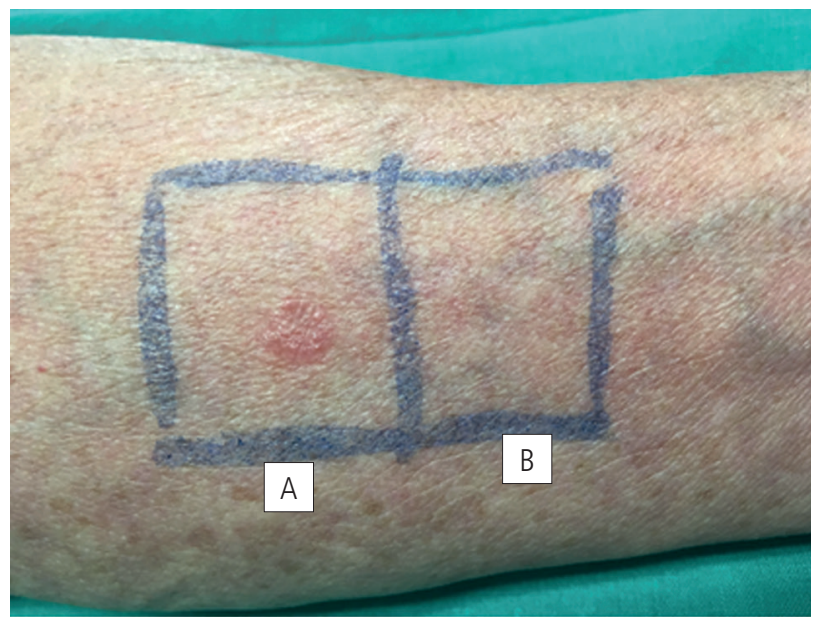

Figure. A, Positive PT with lenalidomide 10\% IN dimethyl sulfoxide. B, Negative result in control test with dimethyl sulfoxide.

spreads the drug well on skin. Petrolatum and water are the most frequently used vehicles, although DMSO may be more adequate for certain drugs. There is no standardized version of skin tests or patch tests with this drug. A single-blind oral challenge test performed with $25 \mathrm{mg}$ of lenalidomide was well tolerated. The drug was prescribed again, and the patient received a further 3 cycles without reactions. During the fourth cycle (6 in total), 3 days later, a generalized erythematous maculopapular exanthema appeared without eosinophilia and was treated with oral antihistamines and corticosteroids. The exanthema resolved in 7 days. Patch testing was repeated. The results were positive with lenalidomide $10 \%$ in DMSO on day $2(++)$ and day $4(++)$. The result of a control test with DMSO was negative (Figure). Similarly, the results of patch testing with lenalidomide were negative in 5 controls.

Since lenalidomide was indispensable for treatment of MDS and the patient had experienced a true delayed hypersensitivity reaction with no eosinophilia or severe clinical signs, we decided to administer the drug in a slow desensitization protocol.

The starting dose was $1 \mathrm{mg}$ administered daily for a week and followed by $2.5 \mathrm{mg}$ daily for a week, then $5 \mathrm{mg}$ daily for a week. On the sixth day of the third week, the patient developed an erythematous maculopapular exanthema on his back, arms, and thorax. No eosinophilia or hepatic or renal involvement was observed. The patient was treated with oral antihistamines and tapering doses of $30 \mathrm{mg}$ of corticosteroids for 5 days until the reaction disappeared. Desensitization was restarted a week after symptoms disappeared, with a reduced dose of lenalidomide (2.5 mg) for another week. Another generalized exanthema appeared on the seventh day, 3 hours after the last dose. The patient was treated with $60 \mathrm{mg}$ of prednisone for 15 days. Corticosteroids were administered in tapering doses for a further 15 days because the symptoms did not remit. There were no residual lesions, desquamation, eosinophilia, or hepatic or renal involvement. At that point, desensitization was discontinued, and another desensitization protocol was proposed, although the patient refused.
We present a case of 2 different clinical patterns in the same patient. It was difficult to distinguish whether these were a cutaneous adverse reaction or hypersensitivity reaction.

To our knowledge, this is the first case of hypersensitivity reaction to lenalidomide confirmed by positive patch test results. Unfortunately, desensitization with lenalidomide was not achieved, probably because the patient's reaction was a true delayed hypersensitivity reaction. All successfully desensitized cases reported in the literature are presumably because of toxicity to the drug and not because of an allergic reaction. Reports of successful desensitization to lenalidomide with similar protocols reveal no positive skin test results or allergology work-up $[1,4,6,8,9]$. Lee et al [1] reported 5 cases of successful slow desensitization to lenalidomide, achieving therapeutic doses of $10 \mathrm{mg}$ after 6 weeks. If $25 \mathrm{mg}$ was needed, the protocol was extended for a further week. As no skin tests were carried out on these patients, it was not possible to confirm the diagnosis. Desensitization to other oral drugs can be achieved in a slow protocol, in contrast with rapid desensitization in the case of immediate hypersensitivity reactions [10]. Cañamares et al [6] reported 2 patients with delayed exanthema after taking lenalidomide and a negative skin test result; both tolerated rapid desensitization.

There are no reports of positive skin test results and successful desensitization to lenalidomide in this kind of delayed reaction [10]. Reported cases occurred mostly after the first cycle and with no positive skin test results, possibly suggesting skin toxicity and thus leading to an erroneous diagnosis of an allergic reaction $[1,4,6,8,9]$. In the case we report, consistent with skin toxicity, the first reaction occurred after 2 cycles, , although the second one appeared after 4 cycles (6 in total), with a positive patch test result. Therefore, a type IV hypersensitivity reaction was confirmed.

It is important to perform a complete allergology work-up in all patients in order to determine the etiologic mechanism of these skin reactions.

In conclusion, skin toxicity due to high doses of lenalidomide is frequent, and true allergic reactions may occur. While our protocol was not successful, the successful desensitization in delayed hypersensitivity reactions reported to date could be because the patients who experienced skin toxicity were not truly allergic patients [10]. More studies are needed to further investigate our findings.

\section{Funding}

The authors declare that no funding was received for the present study.

\section{Conflicts of Interest}

The authors declare that they have no conflicts of interest.

\section{References}

1. Lee $M J$, Wickner $P$, Fanning $L$, Schlossman $R$, Richardson $P$, Laubach J, et al. Lenalidomide desensitization for delayed hypersensitivity reactions in 5 patients with multiple myeloma. Br J Haematol. 2014 Oct;167:127-31. 
2. Nardone B, Wu S, Garden BC, West DP, Reich LM, Lacouture ME. Risk of rash associated with lenalidomide in cancer patients: a systematic review of the literature and metaanalysis. Clin Lymphoma Myeloma Leuk. 2013;13:424-9.

3. Shanbhag A, Pritchard ER, Chatterjee K, Hammond DA. Highly Probable Drug Reaction With Eosinophilia and Systemic Symptoms Syndrome Associated With Lenalidomide. Hosp Pharm. 2017 Jun;52:408-11.

4. Seki JT, Sakurai N, Kukreti V. Lenalidomide Desensitization in Systemic Light-Chain Amyloidosis with Multi-Organ Involvement. J Clin Med Res. 2015 Oct;7:807-11.

5. Escudero-Vilaplana V, Osorio Prendes S, Sanjurjo Sáez M. Suspected itchy rash secondary to lenalidomide. Farm Hosp. 2014 Nov 3;38:495-6.

6. Cañamares Orbis I, García Muñoz C, Cortijo Cascajares S, Méndez Esteban ME. Desensitization to lenalidomide. Farm Hosp. 2012;36:542-55

7. Castaneda CP, Brandenburg NA, Bwire R, Burton GH, Zeldis JB. Erythema multiforme/Stevens-Johnson syndrome/toxic epidermal necrolysis in lenalidomide-treated patients. J Clin Oncol. 2009 Jan 1;27:156-7.

8. Phillips J, Kujawa J, Davis-Lorton M, Hindenburg A. Successful desensitization in a patient with lenalidomide hypersensitivity. Am J Hematol. 2007 Nov;82:1030.

9. Seki JT, Banglawala S, Lentz EM, Reece DE. Desensitization to lenalidomide in a patient with relapsed multiple myeloma. Clin Lymphoma Myeloma Leuk. 2013;13:162-5.

10. Scherer K, Brockow K, Aberer W, Gooi JH, Demoly P, Romano $A$, et al. Desensitization in delayed drug hypersensitivity reactions - an EAACI position paper of the Drug Allergy Interest Group. Allergy. 2013;68:844-52.

Manuscript received October 29, 2018; accepted for publication February 6, 2019.

Blanca Noguerado-Mellado Doctor Esquerdo, 46 28007 Madrid, Spain E-mail: blancanoguerado@gmail.com 